\title{
Selective Modulation of Auditory Cortical Alpha Activity in an Audiovisual Spatial Attention Task
}

\author{
Julia N. Frey, ${ }^{1}$ Nelly Mainy, ${ }^{2}$ Jean-Philippe Lachaux, ${ }^{2}$ Nadia Müller, ${ }^{1}$ Olivier Bertrand, ${ }^{2}$ and Nathan Weisz ${ }^{1}$ \\ ${ }^{1}$ Center for Mind / Brain Sciences, University of Trento, 38068 Rovereto, Italy, and ${ }^{2}$ Brain Dynamics and Cognition Team, Lyon Neuroscience Research \\ Center (CRNL), INSERM U1028, CNRS UMR5292, University Lyon 1, 69500, Lyon-Bron, France
}

Despite substantial research on attentional modulations of visual alpha activity, doubts remain as to the existence and functional relevance of auditory cortical alpha-band oscillations. It has been argued that auditory cortical alpha does not exist, cannot be measured noninvasively, or that it is dependent on visual alpha generators. This study aimed to address these remaining doubts concerning auditory cortical alpha. A magnetoencephalography study was conducted using a combined audiovisual spatial cueing paradigm. In each trial, a cue indicated the side (left or right) and the modality (auditory or visual) to attend, followed by a short lateralized auditory or visual stimulus. Participants were instructed to respond to the stimuli by a button press. Results show that auditory cortical alpha power is selectively modulated by the audiospatial, but not the visuospatial, attention task. These findings provide further evidence for a distinct auditory cortical alpha generator, which can be measured noninvasively.

Key words: alpha; attention; auditory

\section{Introduction}

Alpha oscillations are considered to reflect the excitatory-inhibitory (E-I) balance of sensory and motor areas, with high levels of alpha indicating inhibitory states (Klimesch et al., 2007; Weisz et al., 2007). This view is well established in the visual and sensorimotor domain (Thut and Miniussi, 2009). The first magnetoencephalography (MEG) evidence of an auditory cortical alpha-like rhythm was reported by Lehtelä et al. (1997); however, doubts continue concerning the functional relevance and measurability of an auditory alpha rhythm (Weisz et al., 2011). A concern, mainly based on scalp EEG recordings in audiovisual attention paradigms, is whether noninvasive methods are sensitive enough to measure cortical auditory alpha. In many audiovisual spatial attention tasks, attention is cued to lateralized visual or auditory sensory input, followed by a target stimulus. Studies reported occipitoparietal alpha power increases during auditory attention (Foxe et al., 1998; Fu et al., 2001), interpreted as inhibition of visual processing. Banerjee et al. (2011) offered further insights, reporting modality-independent topographical ("supramodal") effects in early time-windows, and late "modality-specific" topographical differences dependent on attended modality. Although posterior-attentional modulations have been consistently shown

\footnotetext{
Received Nov. 15, 2013; revised Feb. 28, 2014; accepted March 20, 2014.

Author contributions: J.N.F., N. Müller, O.B., and N.W. analyzed data; N. Mainy, J.-P.L., and 0.B. designed research; N. Mainy performed research; J.N.F. and N.W. wrote the paper.

This work was performed within the framework of the Labex Cortex (ANR-11-LABX-0042) of Université de Lyon, within the program "Investissements d'Avenir" (ANR-11-IDEX-0007) operated by the French National Research Agency (ANR), and supported by grants of the Deutsche Forschungsgemeinschaft (We4156/1-1) and the European Research Council (WIN2CON; ERC StG 283404) to N.W.

The authors declare no competing financial interests.

Correspondence should be addressed to Julia Frey, CIMeC, Center for Mind/Brain Sciences, Università degli Studi di Trento, via delle Regole, 101, 38123 Mattarello, TN, Italy. E-mail: julianatascha.frey@unitn.it.

DOI:10.1523/JNEUROSCI.4813-13.2014

Copyright $\odot 2014$ the authors $\quad 0270-6474 / 14 / 346634-06 \$ 15.00 / 0$
}

using scalp EEG, a recent intracranial study by Gomez-Ramirez et al. (2011) showed auditory cortical alpha-band modulations influenced by intersensory attention. This study underlines the general challenge of detecting cognitive auditory cortical alphaband modulations using noninvasive tools (Weisz et al., 2011). However, this has been shown to be feasible. Examples include "tau" desynchronizations (Lehtelä et al., 1997); and working memory dependent modulations of auditory cortical alpha (van Dijk et al., 2010). With regards to attentional manipulations our group has repeatedly demonstrated the sensitivity of MEG, as well as EEG, to capture modulations of auditory cortical alpha (for review, see Weisz and Obleser, 2014). However, in these studies participants had to attend to the auditory modality only. Using a design similar to audiovisual EEG studies, which reported parietal modulations (Banerjee et al., 2011), we set out to demonstrate modality-specific effects of spatial attention on auditory cortical alpha.

We devised a multisensory Posner task (Posner, 1980), in which participants were cued to attend lateralized vision or audition. We predicted alpha effects in occipitoparietal regions independent of the attended modality. Beyond this replication, our main goal was to identify modality specific effects of spatial attention. Source localization would aid in uncovering auditory cortical alpha modulations during audiospatial attention (Weisz et al., 2011). In accordance with these hypotheses, we present here for the first time MEG data of a combined audiospatial and visuospatial attention task showing selective alpha modulations in the auditory cortex only when attention was deployed to the auditory modality.

\section{Materials and Methods}

Participants. Overall 11 healthy participants ( 7 females; mean age, 26.5 years; age range, $22-43$ years) took part in the study after the experimental procedure was explained and written informed consent was obtained. No partic- 


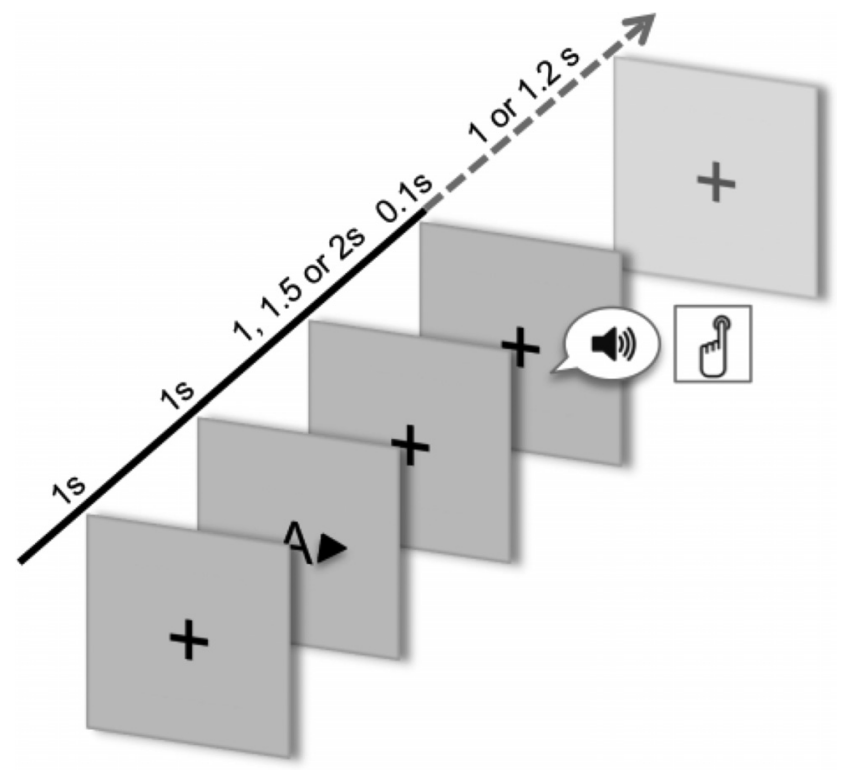

Figure 1. Schematic illustration of a trial. After a fixation cross, a cue indicating the modality (auditory or visual) and side (right or left) is presented, followed by a target stimulus. The figure depicts a valid right auditory trial. Participants were required to press a button as fast as possible after target presentation.

ipant had a history of neurological or psychiatric disorders or hearing/vision impairments. The experimental procedure conformed to the Declaration of Helsinki and was approved by the local ethics committee.

Experimental paradigm. The general outline of a trial is shown in Figure 1. Stimuli were presented with Presentation software (Neurobehavioral Systems). Each trial started with a white cross on a black screen; after $1000 \mathrm{~ms}$ a visual cue indicated the modality (auditory or visual; A or V) and the side (left or right ear/hemifield; $\leftarrow$ or $\rightarrow$ ) of a second stimulus (target). The target was presented after a pseudorandomized interstimulus-interval of 1000,1500 , or $2000 \mathrm{~ms}$. In $80 \%$ of all trials, cues were valid, whereas $20 \%$ of the trials consisted of invalid cues (either concerning modality, side, or both). In the auditory condition, the second stimulus consisted of a noise burst of $100 \mathrm{~ms}$ ( 1 of 5 different pitches), and in the visual condition it consisted of a simple geometric figures (either an upward or downward triangle, a diamond, a circle, or a square). Participants were required to respond to all second stimuli by pressing a button as fast as possible. After 1000 or $1200 \mathrm{~ms}$ a new trial started. There were 6-9 blocks of 60 trials each ( 15 trials with each cue type) resulting in 360-540 trials in total. The whole experiment lasted for $\sim 1.5 \mathrm{~h}$.

Data acquisition. A 275 channel whole-head axial gradiometer system (CTF, VSM MedTech) was used for recording of electromagnetic brain activity, which was sampled continuously at a rate of $600 \mathrm{~Hz}$ (filtered online $0-150 \mathrm{~Hz}$ ). Three coils were placed at nasion and the two preauricular points; passing small currents through these coils before and after each block allowing us to monitor head movements. From each participant we obtained an anatomical 3D MRI, using a 1.5 T Siemens Sonata scanner.

Behavioral data analysis. Task performance was computed with respect to reaction times (RTs), excluding all trials in which no response was given or in which response time was longer than $800 \mathrm{~ms}$ (on average $3.4 \%$ ). Analysis was done using repeated-measures ANOVA with the four factors cue and target modality (auditory or visual), as well as cue and target side (left or right).

Preprocessing. Trials in which no response was given (on average 3.2\%) were excluded from the data analysis. Epochs of $\pm 2000 \mathrm{~ms}$ length were extracted around cue onset resulting in the four cue conditions: auditory right, auditory left, visual right, and visual left. A generous time-interval was chosen to diminish edge effects in the time-frequency estimates. Before an independent component analysis (ICA) to remove eye and heart artifacts, data were detrended, down-sampled to $400 \mathrm{~Hz}$, and visually inspected to remove large muscle artifacts and channel jumps. The ICA was based on 300 randomly selected trials of the concatenated data; and two to five components were rejected in each dataset by visually inspecting the components' time course and topographies. After the ICA, a second visual artifact rejection was done to remove remaining artifacts, resulting in the rejection of $5-46 \%$ of all trials per subject. Following the artifact rejection, trial number across all four conditions was equalized for each participant to prevent any bias in subsequent data analysis across conditions (Gross et al., 2013). This was done by randomly selecting the same number of trials from each condition (i.e., the number of trials present in the condition with the fewest trials) resulting in 72-126 trials per condition and subject.

Sensor level. Spectral estimation (Fourier transformation) was performed on Hanning-tapered time windows from -0.5 to $1.5 \mathrm{~s}$ (in steps of $0.05 \mathrm{~s}$ ) relative to the cue onset from 1 to $30 \mathrm{~Hz}$ (in steps of $1 \mathrm{~Hz}$ ). The length of the sliding time windows was frequency dependent ( 5 cycles of the respective frequency). Postcue neuronal activity was then estimated as a relative power change with respect to a precue time window from -0.5 to $-0.1 \mathrm{~s}$. To test for direction-specific attention effects regardless of the modality, the power of the grand averages of the two auditory and two visual conditions (right vs left) was averaged and statistically compared regarding the time window from 0.3 to 0.9 s postcue and $8-16 \mathrm{~Hz}$ using a nonparametric cluster-based permutation analysis (Maris and Oostenveld, 2007).

To investigate whether there are alpha-band modulations in the auditory cortex in a main-modality contrast, we compared the auditory and visual condition regardless of locus of attention by concatenating all trials of each modality. For both conditions spectral power was estimated from -0.5 precue to 1.5 s postcue (in steps of 0.05 ) for $1-30 \mathrm{~Hz}$ (in steps of 1 $\mathrm{Hz}$; sliding time window: 5 cycles per frequency). The postcue neuronal activity for the subsequent statistical test was estimated as relative power change with respect to the -0.5 to $-0.1 \mathrm{~s}$ precue time window. Then we statistically contrasted the grand averages of the two conditions for the same time-frequency window $(0.3-0.9 \mathrm{~s}, 8-16 \mathrm{~Hz})$ using cluster-based correction for multiple comparisons.

Source level. To localize the probable generators of the sensor level effect, a time-frequency window was defined based on the time courses and power spectra of the respective peak sensors, and a source reconstruction was performed using a dynamic imaging of coherent sources beamformer (DICS; Gross et al., 2001). Cross-spectral density (CSD) matrices of all conditions were calculated using a multitaper FFT method with a center frequency of $11 \pm 3 \mathrm{~Hz}$ for a time period of $0.45-0.9 \mathrm{~s}$ after cue onset. For each subject, realistically shaped, single-shell head models (Nolte, 2003) based on individual structural MRI scans were computed. A grid with $1 \mathrm{~cm}$ resolution was normalized on a MNI template, and then morphed into the brain volume of each participant. Leadfields for all grid points and a CSD matrix based on the concatenated auditory and visual conditions (right and left) were used to compute a common spatial filter for each grid point and each participant. Using this common filter, the spatial distribution of power was estimated for all four conditions (modality $\times$ side). Dependent-sample $t$ tests were calculated between the attention-direction (right vs left) in both modalities using the grand averages. Additionally, a conjunction/disjunction analysis based on the statistical maps of the source analysis was performed to illustrate modality-independent and -specific activation. In this way, regions active in both modalities (auditory and visual; conjunction) as well as regions specific to the auditory modality [auditory - (auditory and visual); auditory disjunction] and to the visual modality [visual - (auditory and visual); visual disjunction] were identified.

Additionally, to specifically investigate whether the alpha-band in the auditory cortex is modulated in the main modality contrast (auditory vs visual), we performed a second DICS beamformer analysis using all trials from the auditory and the visual condition, collapsed across spatial attention. Based on the sensor-level data, spatial filters were estimated for the $0.5-1 \mathrm{~s}$ time- and $10 \pm 3 \mathrm{~Hz}$ frequency-window. All other parameters were identical to the first beamformer analysis described above. In a second approach, all parameters (also the time-frequency window: 0.45 $0.9 \mathrm{~s}, 11 \pm 3 \mathrm{~Hz}$ ) were identical to the beamformer analysis described above. Because the main outcomes were virtually identical, in the present paper we restrict our description to the first approach. All offline treat- 
A
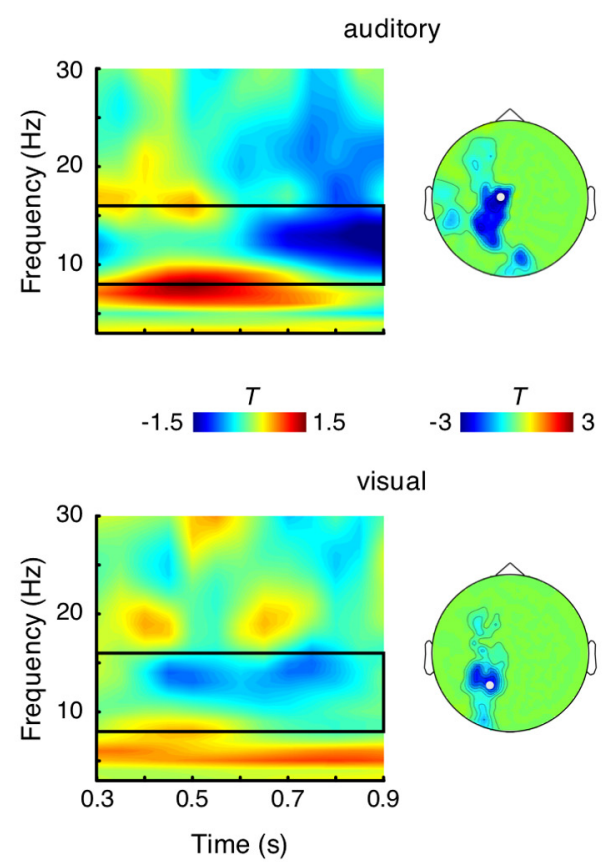

B

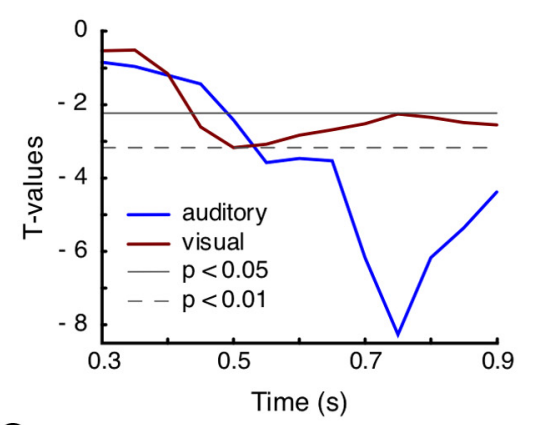

C

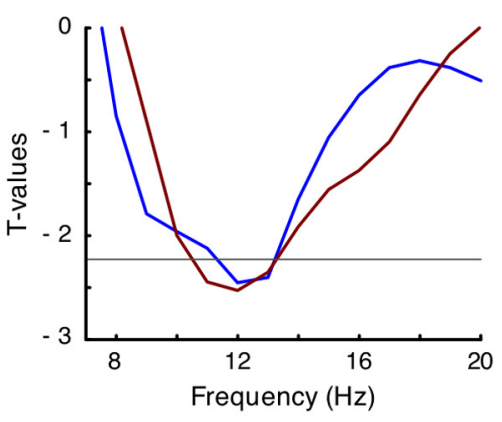

D

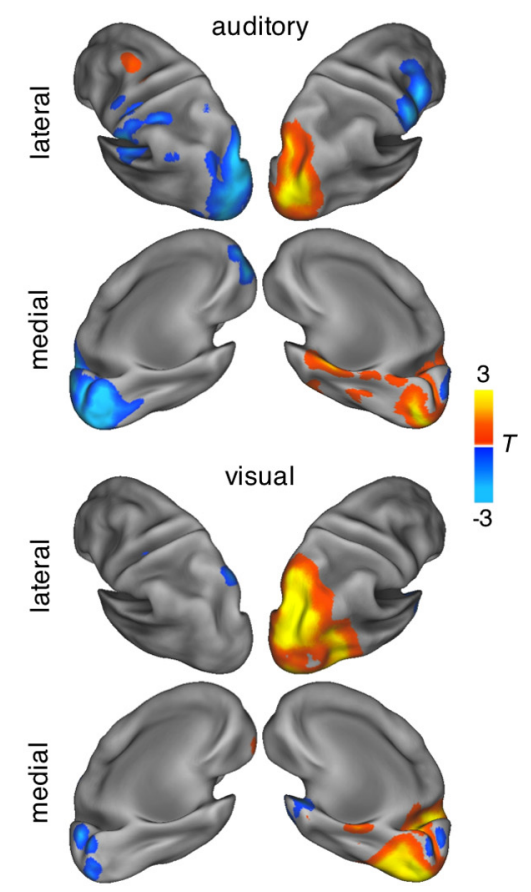

Figure 2. Modality-specific effects. Results of the contrast attend right versus attend left in the auditory and visual condition (positive and negative values refer to relative power increases and decreases, respectively). $\boldsymbol{A}$, Time-frequency plots and sensor-level topographies (masked at $p<0.05$; corrected for multiple comparisons) showing the statistical contrasts attend right versus attend left for 300-900 ms post-cue and averaged across $8-16 \mathrm{~Hz}$ (marked with a solid black rectangle) in the auditory condition (top, $p=0.001$ ) and visual condition (bottom, $p=0.026$ ). $C$, Time course and power spectrum of the auditory and visual contrasts (shown are the statistical values of the peak channels, marked with a white dot in A). $\boldsymbol{D}$, Statistical contrast attend right versus left (uncorrected) of the source reconstructions of the auditory (top) and visual conditions (bottom) for the $450-900$ ms postcue time- and $11 \pm 3 \mathrm{~Hz}$ frequency-window (masked at $p=0.05$ ).

ment of MEG data were performed using fieldtrip (http://fieldtrip. fcdonders.nl/; Oostenveld et al., 2011).

\section{Results}

\section{Cue modality and cue side influence reaction time of} target detection

An ANOVA with the four factors cue modality, cue side, target modality, and target side was applied to RTs. A main effect for the target modality was observed $\left(F_{(1,10)}=439.44, p<0.001\right)$, with shorter mean RTs for auditory than visual targets. In addition, the allocation of attentional resources to the cued modality/side produced typical cost-benefit patterns, resulting in significant interactions between the cue and the target modality $\left(F_{(1,10)}=48.28\right.$, $p<0.001)$ as well as the cue and the target side $\left(F_{(1,10)}=44.48\right.$, $p<0.001)$. The effect of the cue-target modality interaction was particularly pronounced when an auditory cue was followed by a visual target due to the invalid cueing and generally longer reaction times for visual targets. Furthermore, we found a significant interaction between the cue side and the target modality $\left(F_{(1,10)}=\right.$ 12.44, $p<0.01)$.

\section{Postcue alpha effects in audiospatial and visuospatial attention: sensor level}

Nonparametric statistical contrasts in the auditory and the visual modality (attend right vs attend left) regarding the timefrequency window from $0.3-0.9 \mathrm{~s}$ after cue onset and $8-16 \mathrm{~Hz}$ (see Materials and Methods) resulted in significant negative auditory $(p<0.001)$ and visual $(p=0.026)$ clusters emerging at 0.5 and $0.45 \mathrm{~s}$ postcue, respectively (Fig. $2 A, B$ ). On a descriptive level, the topographies of both clusters were left-lateralized and strongest at parietal sensors. However, the auditory cluster spread to more posterior, and to left temporal and frontal sen- sors, whereas the visual cluster already emerged more posteriorly, with some later activation in left frontal sensors. The spectral profile of the peak channel of the auditory and the visual cluster indicated the effect to be centered $\sim 11 \mathrm{~Hz}$ (Fig. 2C).

\section{Postcue alpha effects in audiospatial and visuospatial attention: source level}

To localize probable generators contributing to the sensor-level effect, we performed a beamformer analysis and subsequent $t$ tests on the source level data contrasting attend right versus attend left (see Materials and Methods). Note in the following sections that alpha modulations are described as "relative", as they refer to a contrast, which does not provide conclusive information in which condition the alpha oscillations were modulated (e.g., increase for attend right, decrease for attend left, or both). In the auditory condition, we found relative alpha power decreases in the left auditory cortex (BA40-42 and postcentral gyrus), in the left primary, secondary, and associative visual cortices (BA17-19), the left superior frontal gyrus (BA11), and in the right inferior frontal and precentral gyri (BA44, BA6) when attention was directed to the right compared with the left side (Fig. $2 D$; positive and negative values refer to relative power increases and decreases, respectively). Additionally, when attending the right side, relative alpha power increases were identified in the left middle frontal gyrus (BA6) and right occipitoparietal regions (BA17, superior parietal lobule). Interestingly, no modulations of spatial attention were found in the right auditory cortex.

In the visual condition, when the right side was attended compared with the left side, we found small relative alpha power decreases in the left primary visual area (BA17), the left superior parietal lobule (BA7), and the right superior temporal gyrus 

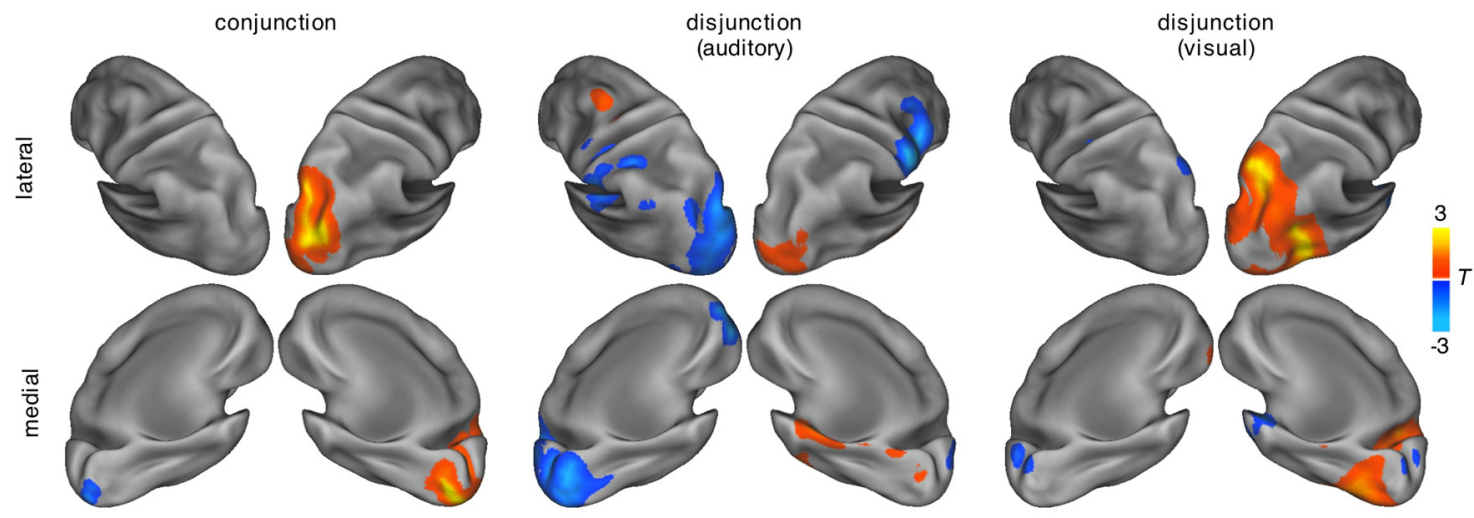

Figure 3. Results of the conjunction analysis based on the statistical maps of the source analysis (shown in Fig. 2D). Shown are source activity of the contrast attend right versus attend left common to both conditions (left), and specific to the auditory (middle), and the visual condition (right; positive and negative values refer to relative power increases and decreases, respectively).

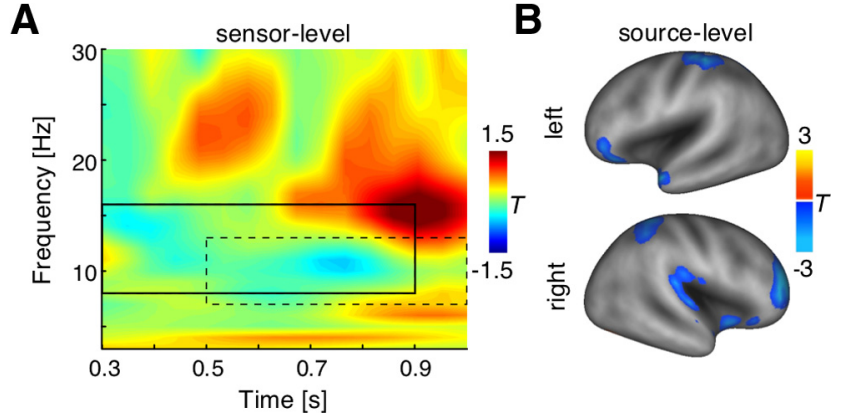

Figure 4. Main modality effects. $A$, Time-frequency data from 3 to $30 \mathrm{~Hz}$ and $300-1000 \mathrm{~ms}$ postcue, showing the contrast between auditory and visual modalities. A nonparametric statistical test (cluster-based correction for multiple comparisons) was computed for the time-frequency window of $300-900 \mathrm{~ms}$ and $8-16 \mathrm{~Hz}$ (marked with a solid black rectangle). B, Statistical contrast of the source reconstruction of the auditory and the visual condition using the time-frequency window of $500-1000 \mathrm{~ms}$ and $10 \pm 3 \mathrm{~Hz}$ (marked in $A$ with a dotted black rectangle). Shown are the lateral views (left and right) of inflated hemispheres thresholded at $p=0.05$ (uncorrected).

(BA38). Similar to the auditory contrast, there were relative alpha power increases in the right occipitoparietal areas when attend right versus attend left. However, these increases were more extensive than in the auditory contrast including right primary, secondary and associative visual cortices (BA17-19), parts of the right fusiform gyrus (BA37), and the right superior parietal lobule (BA7; Fig. 2D). The conjunction analysis showed that relative alpha power decreases in the right primary and associative visual cortices (BA17, BA19), and the right superior parietal lobule (BA7), were common to both the auditory and the visual condition. Importantly, the auditory disjunction analysis clearly illustrated that the left auditory cortical alpha was solely modulated when attention was directed at acoustic input (Fig. 3).

Postcue main modality alpha effects: sensor and source level On a descriptive level, the contrast between auditory and visual attention (in the $0.5-1 \mathrm{~s}$ time- and $8-16 \mathrm{~Hz}$ frequency-window) resulted in a negative pattern conforming to our expectations, however, without reaching statistical significance $(p=0.53$; Fig. $4 A$ ). However, as outlined above circumscribed auditory cortical effects can be missed in the sensor level analysis especially when using cluster level correction (which favors broadly spread effects). Therefore, targeting the alpha frequency range (see Materials and Methods) a DICS was performed to scrutinize this issue in more detail. Indeed, the source reconstruction of the modality contrast shows an alphaband modulation in the right auditory cortex (BA 41); however, interestingly, no similar modulation was found in the left auditory cortex (Fig. 4B). Furthermore, some additional modulations were found in bilateral parietal regions (postcentral gyri, right superior parietal lobule), frontal regions (right superior frontal gyrus, bilateral middle frontal gyri), and bilateral temporal regions (left temporal pole, right insular cortex). Also, in contrast to the spatial attention effect described in Figures 2 and 3, occipital effects were basically absent in the modality contrast.

\section{Discussion}

Audiospatial attention modulates cortical auditory alpha

For the first time we present noninvasively measured auditory alpha power modulations in an audiovisual attention paradigm. Audiospatial attention resulted in alpha power modulations in the left auditory cortex (and not in the right auditory cortex), whereas visuospatial attention mainly modulated alpha in visual areas, but not in the auditory cortex. Alpha band oscillations are thought to be a general mechanism regulating the excitability state of neural tissue (Klimesch et al., 2007) and a top-down driven mechanism to disengage cortical areas in stimulus processing (Jensen and Mazaheri, 2010). Importantly however, two potential mechanisms could influence auditory processing during an audiospatial attention task. On the one hand, processing of the unattended ear, mainly in the auditory cortex ipsilateral to attention, could be inhibited. On the other hand, processing of the attended ear, mainly in the hemisphere contralateral to attention, could be facilitated (Tervaniemi and Hugdahl, 2003). The statistical contrast reported in this study, however, does not disclose which mechanism was driving our effects (ipsilateral inhibition, contralateral facilitation or both). The present alpha modulations in the left auditory cortex could, therefore, reflect an inhibition of this region when the left ear was attended, or an increased excitability state of this region when the right ear was attended (or both). These results are in line with findings from the other modalities, in which relative alpha power increases were found in to-be-inhibited areas in visuospatial (Worden et al., 2000; Sauseng et al., 2005; Thut et al., 2006), visual feature-based (Snyder and Foxe, 2010), and somatosensory attention tasks (Haegens et al., 2011). Due to several difficulties in the study of cortical auditory alpha oscillations, particularly with scalp EEG recordings (e.g., the size and orientation of the auditory cortex, the blurring by simultaneous somatosensory and visual alpha fluctuations), it was either thought that the alpha-band modulations in auditory spatial attention studies resulted from posterior alpha generators, or that the increased sensitivity of intracranial recordings is necessary to measure them (Gomez-Ramirez et al., 
2011). Using a very similar design as in aforementioned audiovisual studies (Foxe et al., 1998; Worden et al., 2000; Fu et al., 2001; Banerjee et al., 2011; Gomez-Ramirez et al., 2011), the current MEG study, however, clearly shows alpha-band modulations generated in the auditory cortex corroborating findings from previous research in the auditory modality (Weisz et al., 2011; Weisz et al., 2013; Müller and Weisz, 2012). These findings contribute to the field of auditory cognition on several levels. First, they provide more evidence for the existence of auditory alpha and that it can be measured with noninvasive techniques. Second, they show that its generation is separate from occipital alphaband generators. Finally, they support the notion that auditory alpha is functionally relevant in auditory cognition, here in audiospatial attention; corroborating the notion that alpha possesses general similar functional properties.

\section{Audiospatial attention is processed asymmetrically}

When attending the right versus the left side alpha-band oscillations were modulated in the left but not the right auditory cortex. A reason for these results could be the asymmetries of the auditory system. Whereas the left auditory cortex mainly localizes stimuli in the contralateral space, the right auditory cortex processes stimuli in the whole space (Zatorre and Penhune, 2001; Spierer et al., 2009). Subsequently, there could have been similar alpha-band modulations in the right auditory cortex during audiospatial attention to the left and to the right side. Indeed, alphaband oscillations in the right but not in the left auditory cortex are modulated when contrasting auditory versus visual attention regardless of locus of attention (Gomez-Ramirez et al., 2011). These results from the modality-specific audiospatial contrast and the main modality contrast are in line with previous results from our group (Müller and Weisz, 2012; Weisz et al., 2013), providing further evidence for asymmetries in the auditory system during audiospatial attention before stimulus onset.

\section{Audiospatial attention modulates alpha outside of the auditory cortex}

We hypothesized that spatial attention, regardless of modality, modulates alpha-band activity in posterior regions, reflecting a supramodal attention system (Banerjee et al., 2011). However, in addition to these common modulations, we report posterior alpha-band activity modulated by audiospatial but not visuospatial attention. It is worth noting that these modulations, in contrast to modulations in the auditory system, were symmetrical. As the current study focused on spatial attention within one modality, and as there was no simultaneous, to-be-ignored visual input during audiospatial attention, it is unlikely that these findings reflect a general disengagement of the visual system. On the contrary, they could reflect an automatic coactivation of visual areas during audiospatial attention either because the visual cue enhanced the salience of the spatial location regardless of the modality (Smith and Schenk, 2012), or because the visual and the auditory systems actively interact during audiospatial attention. Interestingly however on a behavioral level, dramatic costs in RTs were obtained when participants were wrongly cued to the auditory modality (i.e., a visual target appeared), meaning that this coactivation is not beneficial in a functional sense. Alternatively, also taking into account alpha power modulations in the posterior parietal cortex and the inferior and middle frontal gyri, these changes could reflect involvement of the dorsal and ventral attention networks (Corbetta and Shulman, 2002; Petersen and Posner, 2012; Wen et al., 2012). Involvement of both attention networks has been found in audiospatial attention tasks using fMRI and MEG (Degerman et al., 2006; Weisz et al., 2013;
Kong et al., 2014; Lee et al., 2014). Together, the alpha-band modulations in regions outside of the auditory cortex could either reflect a coactivation of visual areas due to visual dominance or audiovisual interactions, or an involvement of the dorsal and ventral attentional networks.

\section{Common posterior alpha-band modulations}

The present study yielded interesting results concerning attention deployment across modalities in line with our hypotheses. The conjunction analysis showed that audiospatial as well as visuospatial attention modulated alpha-band oscillations mainly in right posterior areas. This activation could partly reflect automatic activation of visual areas during audiospatial attention (see above); however, it could also reflect a supramodal attentional system (Banerjee et al., 2011) or an interactive synergy between the auditory and the visual modality. The posterior parietal cortex has been suggested to play a central role in multisensory integration (Macaluso et al., 2003; Molholm et al., 2005) including auditory spatial attention (Wu et al., 2007; Hill and Miller, 2010; Smith et al., 2010, Banerjee et al., 2011). Additionally, the dorsal attention network, including parietal regions, has been suggested to be involved in visuospatial (Kastner and Ungerleider, 2000; Corbetta and Shulman, 2002; Serences and Yantis, 2007; Capotosto et al., 2009) and audiospatial attention tasks (Degerman et al., 2006; Mayer et al., 2006; Shomstein and Yantis, 2006; Wu et al., 2007; Salmi et al., 2009; Lee et al., 2014; Weisz and Obleser, 2014). The present data are in line with these studies suggesting a parietal supramodal attention system (Banerjee et al., 2011; Ptak, 2012; Kong et al., 2014). Alternatively, neural substrates common to audiospatial and visuospatial attention could also represent an interactive synergy improving task performance in both or particularly the auditory modality.

\section{Limitations}

There are some limitations of the current design. First, the cues were presented visually in the audiospatial as well as the visuospatial attention task. To study the effects of attentional cues without any confounds of the cue modality, one could present them in a modality different from the target modalities. However, previous studies have used cues in the same modality as the target (Worden et al., 2000; Sauseng et al., 2005) as well as in a different modality (Fu et al., 2001; Thut et al., 2006; Haegens et al., 2011) showing no fundamental differences of cue modality on attentional effects. Also for our purposes of studying auditory cortical alpha, use of a visual cue poses no interpretational problems. Second, because alpha power is modulated by cue validity (Haegens et al., 2011), the observed effects could have been stronger and possibly clearer if all cues had been valid.

\section{Conclusion}

In accordance with the hypotheses, the present study has shown very selective noninvasively measured alpha-band modulations in the auditory cortex during audiospatial attention. Furthermore, visuospatial attention modulated alpha oscillations in visual areas but not in the auditory cortex, and both audiospatial and visuospatial attention resulted in effects in higher-order areas. These findings provide clear evidence that auditory cortical alpha modulations can be measured noninvasively and that there is a distinct alpha generator in the auditory cortex separate from occipital alpha generators. In addition, they support the notion that alpha-band oscillations possess general functional properties that are also relevant in auditory cognition. 


\section{References}

Banerjee S, Snyder AC, Molholm S, Foxe JJ (2011) Oscillatory alpha-band mechanisms and the deployment of spatial attention to anticipated auditory and visual target locations: supramodal or sensory-specific control mechanisms? J Neurosci 31:9923-9932. CrossRef Medline

Capotosto P, Babiloni C, Romani GL, Corbetta M (2009) Frontoparietal cortex controls spatial attention through modulation of anticipatory alpha rhythms. J Neurosci 29:5863-5872. CrossRef Medline

Corbetta M, Shulman GL (2002) Control of goal-directed and stimulus-driven attention in the brain. Nat Rev Neurosci 3:201-215. CrossRef Medline

Degerman A, Rinne T, Salmi J, Salonen O, Alho K (2006) Selective attention to sound location or pitch studied with fMRI. Brain Res 1077:123-134. CrossRef Medline

Foxe JJ, Simpson GV, Ahlfors SP (1998) Parieto-occipital $\sim 10 \mathrm{~Hz}$ activity reflects anticipatory state of visual attention mechanisms. Neuroreport 9:3929-3933. CrossRef Medline

Fu KM, Foxe JJ, Murray MM, Higgins BA, Javitt DC, Schroeder CE (2001) Attention-dependent suppression of distracter visual input can be crossmodally cued as indexed by anticipatory parieto-occipital alpha-band oscillations. Brain Res Cogn Brain Res 12:145-152. CrossRef Medline

Gomez-Ramirez M, Kelly SP, Molholm S, Sehatpour P, Schwartz TH, Foxe JJ (2011) Oscillatory sensory selection mechanisms during intersensory attention to rhythmic auditory and visual inputs: a human electrocorticographic investigation. J Neurosci 31:18556-18567. CrossRef Medline

Gross J, Kujala J, Hamalainen M, Timmermann L, Schnitzler A, Salmelin R (2001) Dynamic imaging of coherent sources: studying neural interactions in the human brain. Proc Natl Acad Sci U S A 98:694-699. CrossRef Medline

Gross J, Baillet S, Barnes GR, Henson RN, Hillebrand A, Jensen O, Jerbi K, Litvak V, Maess B, Oostenveld R, Parkkonen L, Taylor JR, van Wassenhove V, Wibral M, Schoffelen JM (2013) Good practice for conducting and reporting MEG research. Neuroimage 65:349-363. CrossRef Medline

Haegens S, Händel BF, Jensen O (2011) Top-down controlled alpha band activity in somatosensory areas determines behavioral performance in a discrimination task. J Neurosci 31:5197-5204. CrossRef Medline

Hill KT, Miller LM (2010) Auditory attentional control and selection during cocktail party listening. Cereb cortex 20:583-590. CrossRef Medline

Jensen O, Mazaheri A (2010) Shaping functional architecture by oscillatory alpha activity: gating by inhibition. Front Hum Neurosci 4:186. CrossRef Medline

Kastner S, Ungerleider LG (2000) Mechanisms of visual attention in the human cortex. Annu Rev Neurosci 23:315-341. CrossRef Medline

Klimesch W, Sauseng P, Hanslmayr S (2007) EEG alpha oscillations: the inhibition-timing hypothesis. Brain Res Rev 53:63-88. CrossRef Medline

Kong L, Michalka SW, Rosen ML, Sheremata SL, Swisher JD, ShinnCunningham BG, Somers DC (2014) Auditory spatial attention representations in the human cereb cortex. Cereb Cortex 24:773-784. CrossRef Medline

Lee AK, Larson E, Maddox RK, Shinn-Cunningham BG (2014) Using neuroimaging to understand the cortical mechanisms of auditory selective attention. Hear Res 307:111-120. CrossRef Medline

Lehtelä L, Salmelin R, Hari R (1997) Evidence for reactive magnetic 10-Hz rhythm in the human auditory cortex. Neurosci Lett 222:111-114. CrossRef Medline

Macaluso E, Driver J, Frith CD (2003) Multimodal spatial representations engaged in human parietal cortex during both saccadic and manual spatial orienting. Curr Biol 13:990-999. CrossRef Medline

Maris E, Oostenveld R (2007) Nonparametric statistical testing of EEG- and MEG-data. J Neurosci Methods 164:177-190. CrossRef Medline

Mayer AR, Harrington D, Adair JC, Lee R (2006) The neural networks underlying endogenous auditory covert orienting and reorienting. Neuroimage 30:938-949. CrossRef Medline

Molholm S, Martinez A, Ritter W, Javitt DC, Foxe JJ (2005) The neural circuitry of pre-attentive auditory change-detection: an fMRI study of pitch and duration mismatch negativity generators. Cereb Cortex 15:545551. CrossRef Medline

Müller N, Weisz N (2012) Lateralized auditory cortical alpha band activity and interregional connectivity pattern reflect anticipation of target sounds. Cereb cortex 22:1604-1613. CrossRef Medline

Nolte G (2003) The magnetic lead field theorem in the quasi-static approximation and its use for magnetoencephalography forward calculation in realistic volume conductors. Phys Med Biol 48:3637-3652. CrossRef Medline

Oostenveld R, Fries P, Maris E, Schoffelen JM (2011) FieldTrip: Open source software for advanced analysis of MEG, EEG, and invasive electrophysiological data. Comput Intell Neurosci 2011:156869. CrossRef Medline

Petersen SE, Posner MI (2012) The attention system of the human brain: 20 years after. Annu Rev Neurosci 35:73-89. CrossRef Medline

Posner MI (1980) Orienting of attention. Q J Exp Psychol 32:3-25. CrossRef Medline

Ptak R (2012) The frontoparietal attention network of the human brain: action, saliency, and a priority map of the environment. Neuroscientist 18:502-515. CrossRef Medline

Salmi J, Rinne T, Koistinen S, Salonen O, Alho K (2009) Brain networks of bottom-up triggered and top-down controlled shifting of auditory attention. Brain Res 1286:155-164. CrossRef Medline

Sauseng P, Klimesch W, Stadler W, Schabus M, Doppelmayr M, Hanslmayr S, Gruber WR, Birbaumer N (2005) A shift of visual spatial attention is selectively associated with human EEG alpha activity. Eur J Neurosci 22:2917-2926. CrossRef Medline

Serences JT, Yantis S (2007) Spatially selective representations of voluntary and stimulus-driven attentional priority in human occipital, parietal, and frontal cortex. Cereb Cortex 17:284-293. CrossRef Medline

Shomstein S, Yantis S (2006) Parietal cortex mediates voluntary control of spatial and nonspatial auditory attention. J Neurosci 26:435-439. CrossRef Medline

Smith DT, Schenk T (2012) The premotor theory of attention: time to move on? Neuropsychologia 50:1104-1114. CrossRef Medline

Smith DV, Davis B, Niu K, Healy EW, Bonilha L, Fridriksson J, Morgan PS, Rorden C (2010) Spatial attention evokes similar activation patterns for visual and auditory stimuli. J Cogn Neurosci 22:347-361. CrossRef Medline

Snyder AC, Foxe JJ (2010) Anticipatory attentional suppression of visual features indexed by oscillatory alpha-band power increases: a high-density electrical mapping study. J Neurosci 30:4024-4032. CrossRef Medline

Spierer L, Bellmann-Thiran A, Maeder P, Murray MM, Clarke S (2009) Hemispheric competence for auditory spatial representation. Brain 132: 1953-1966. CrossRef Medline

Tervaniemi M, Hugdahl K (2003) Lateralization of auditory-cortex functions. Brain Res Rev 43:231-246. CrossRef Medline

Thut G, Miniussi C (2009) New insights into rhythmic brain activity from TMS-EEG studies. Trends Cogn Sci 13:182-189. CrossRef Medline

Thut G, Nietzel A, Brandt SA, Pascual-Leone A (2006) Alpha-band electroencephalographic activity over occipital cortex indexes visuospatial attention bias and predicts visual target detection. J Neurosci 26:9494-9502. CrossRef Medline

van Dijk H, Nieuwenhuis IL, Jensen O (2010) Left temporal alpha band activity increases during working memory retention of pitches. Eur J Neurosci 31:1701-1707. CrossRef Medline

Weisz N, Obleser J (2014) Synchronisation signatures in the listening brain: a perspective from non-invasive neuroelectrophysiology. Hear Res 307: 16-28. CrossRef Medline

Weisz N, Müller S, Schlee W, Dohrmann K, Hartmann T, Elbert T (2007) The neural code of auditory phantom perception. J Neurosci 27:14791484. CrossRef Medline

Weisz N, Hartmann T, Müller N, Lorenz I, Obleser J (2011) Alpha rhythms in audition: cognitive and clinical perspectives. Front Psychol 2:73. CrossRef Medline

Weisz N, Müller N, Jatzev S, Bertrand O (2013) Oscillatory alpha modulations in right auditory regions reflect the validity of acoustic cues in an auditory spatial attention task. Cereb Cortex, in press. CrossRef Medline

Wen X, Yao L, Liu Y, Ding M (2012) Causal interactions in attention networks predict behavioral performance. J Neurosci 32:1284-1292. CrossRef Medline

Worden MS, Foxe JJ, Wang N, Simpson GV (2000) Anticipatory biasing of visuospatial attention indexed by retinotopically specific alpha-band electroencephalography increases over occipital cortex. J Neurosci 20:RC63. Medline

Wu CT, Weissman D, Roberts KC, Woldorff MG (2007) The neural circuitry underlying the executive control of auditory spatial attention. Brain Res 1134:187-198. CrossRef Medline

Zatorre RJ, Penhune VB (2001) Spatial localization after excision of human auditory cortex. J Neurosci 21:6321-6328. Medline 